Pacific Journal of Mathematics

A THEOREM ON PARTITIONS OF MASS-DISTRIBUTION 


\title{
A THEOREM ON PARTITIONS OF MASS-DISTRIBUTION
}

\author{
V. V. MENON
}

\begin{abstract}
A 'bisector' of a continuous mass-distribution $M$ in a bounded region on the plane is defined as a straight line such that the two half-planes determined by this line contain half the mass of $M$ each. It is known that there exists at least one point (in the plane) through which pass three bisectors of $M$.
\end{abstract}

Theorem. Let, for a continuous mass distribution $M$, the point $P$ through which three bisectors pass be unique. Then all bisectors of $M$ pass through $p$.

The following corollary also is established: For a convex figure $K$ (i.e., compact convex set with nonempty interior) to be centrally symmetric, it is necessary and sufficient that the point through which three bisectors of area pass be unique.

In what follows, $M$ stands for any continuous mass-distribution in a compact domain in the plane. A line $l$ is called a bisector of $M$ if the two half-planes determined by $l$ contain equal masses of $M$.

The following results are well-known regarding bisectors of $M$. (see, for example, [4], Problem 3-1, 3-2, and [1]).

(1) Let $l$ be any line in the plane. There is a bisector of $M$ parallel to $l$.

(2) There exists a point $P$ in the plane and two perpendicular lines through $P$ such that the portions of $M$ contained in each of the four 'wedges' determined by these lines have the same mass, namely, a quarter of that of $M$.

(3) There exists a point in the plane through which three distinct bisectors of $M$ pass.

Further, let $l_{0}$ be a bisector of $M$ and 0 a fixed point on $l_{0}$. Let $l(\alpha)$ be a bisector of $M$, inclined to $l_{0}$ at an angle $\alpha$ and intersecting $l_{0}$ in $P_{\alpha}$. It is easy to verify that we can choose the bisector $l(\alpha)$ such that the distance $0 P_{\alpha}$ is a continuous function of $\alpha$. We shall make use of this observation in the following.

In this paper we shall investigate the nature of the points through which three distinct bisectors of $M$ pass. Specifically, let $P$ be a point

Received January 25, 1964, and in revised form July 30, 1964. 
in the plane such that three distinct bisectors $l_{1}, l_{2}, l_{3}$ of $M$ pass through $P$; and let $l(\alpha)$ be a bisector of $M$ not passing through $P$. We shall prove the existence of a point $P_{1} \neq P$ such that three distinct bisectors pass through $P_{1}$ too.

First, let $l(\alpha)$ be parallel to one of $l_{1}, l_{2}, l_{3}$; say, to $l_{1}$. Since $l_{1}$ and $l(\alpha)$ are both bisectors, it follows that the portion of $M$ contained between these lines $l_{1}$ and $l(\alpha)$ has zero mass (see Fig. 1).

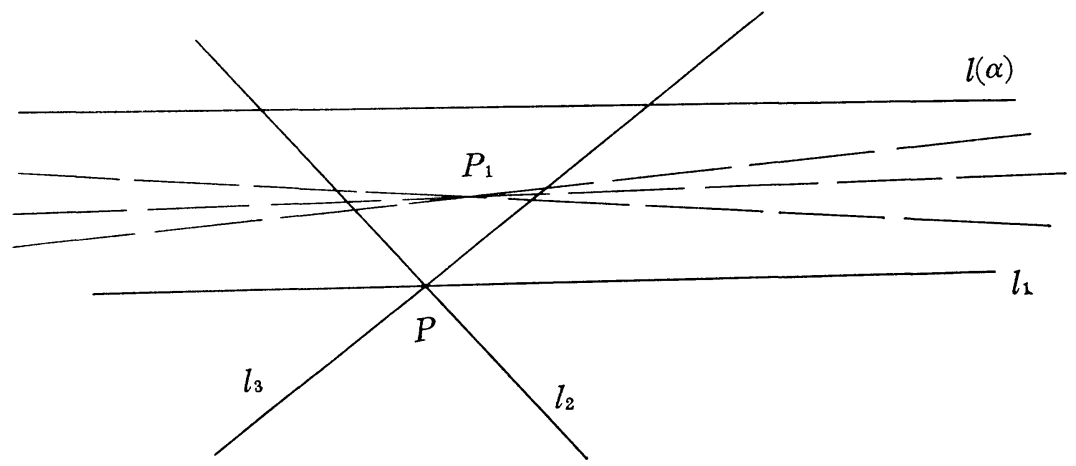

Fig. 1

Since $M$ is enclosed in a bounded domain $D$, we can choose a point $P_{1}$ midway between $l_{1}$ and $l_{(\alpha)}$, and three distinct lines through $P_{1}$ such that each of these three lines intersects (if at all) $l_{1}$ and $l_{(\alpha)}$ outside $D$. In other words, these three lines are bisectors of $M$.

Secondly, let $l_{(\alpha)}$ intersect the lines $l_{1}, l_{2}, l_{3}$ (see Fig. 2), and let $X$ be the point of intersection of $l_{(\alpha)}$ with $l_{2}$. (We number the lines $l_{1}, l_{2}, l_{3}$, such that $X$ lies between the points of intersection of $l_{(\alpha)}$ with $l_{1}$ and $l_{3}$ ).

With reference to a fixed direction, let $\theta_{1}$ and $\theta_{2}$ be the directions of $l_{1}$ and $l_{3}$ respectively, and let $\alpha$ be that of $l_{(\alpha)}$.

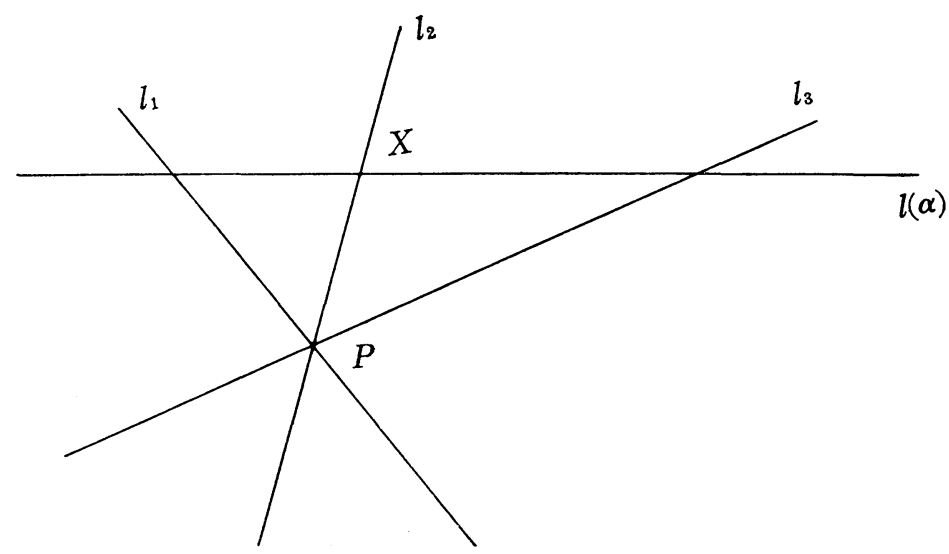

Fig. 2 
When $\theta$ varies from $\theta_{1}$ to $\theta_{2}$ we can choose the bisectors $l(\theta)$ such that $l\left(\theta_{1}\right)=l_{1}, l\left(\theta_{2}\right)=l_{3}$, and $P X=x(\theta)$ is a continuous function of $\theta$. (The equality $l\left(\theta_{1}\right)=l_{1}$ means that the lines $l\left(\theta_{1}\right)$ and $l_{1}$ coincide).

Since $x\left(\theta_{1}\right)=0=x\left(\theta_{2}\right)$, and for the given bisector $\lambda(\alpha), x(\alpha) \neq 0$, if follows that there are two distinct values $\alpha_{1}$ and $\alpha_{2}$ for which

$$
x\left(\alpha_{1}\right)=x\left(\alpha_{2}\right) \neq 0 \text {. }
$$

Let $P_{1}$ be the position of $X$ corresponding to $x\left(\alpha_{1}\right)$. Thus three distinct bisectors $l_{2}, l\left(\alpha_{1}\right)$ and $l\left(\alpha_{2}\right)$ pass through $P_{1}$, and $P_{1} \neq P$.

This proves the required assertion, that is, if a bisector $r$ of $M$ does not pass through $P$ and three distinct bisectors pass through $P$ then there is a point $P_{1}$ distinct from $P$ through which also pass three distinct bisectors.

Hence we have the

THEOREM. Let, for a continuous mass distribution $M$, the point $P$ through which three distinct bisectors of $M$ pass be unique. Then all bisectors of $M$ pass through $P$. (In particular, every line through $P$ bisects $M)$.

Something more can be asserted about the mass distribution $M$ in the following special case. Consider a compact convex figure $K$ (i.e., a compact convex set with nonempty interior) and interpret mass as the area. Since the bisector in any direction is unique, it follows from the above theorem that every line through $P$ is bisector of $K$ where $P$ is the unique point through which three bisectors pass. Consider two such bisectors inclined at a small angle $\theta$, as in Figure 3.

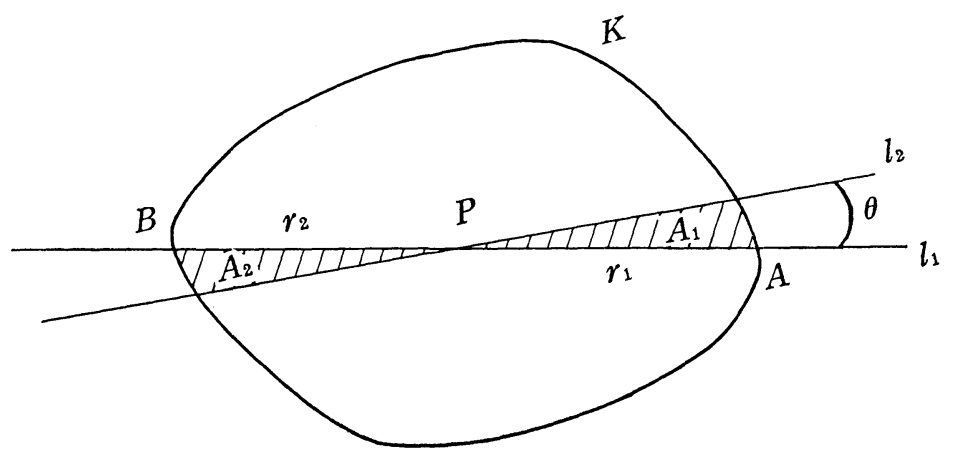

Fig. 3

Let $l_{1}$ intersect the boundary of $K$ in $A$ and $B$, and let $P A=r_{1}$, $P B=r_{2}$ Denote by $A_{1}$ and $A_{2}$, respectively, the areas of the portions of $K$ in the two wedges (shaded in the figure) between $l_{1}, l_{2}$. We have, for small $\theta$, the approximate equalities 


$$
\begin{aligned}
& A_{1} \doteq \frac{1}{2} r_{1}^{2} \theta \\
& A_{2} \doteq \frac{1}{2} r_{2}^{2} \theta
\end{aligned}
$$

$A_{1}=A_{2}$ since $l_{1}, l_{2}$ are both bisectors, and hence $r_{1}=r_{2}$ by making $\theta$ approach zero. As this is true for any position of $l_{1}$, it follows that the figure $K$ is centrally symmetric and $P$ is its centre.

Of course, the converse also is true because any line through the centre of any centrally symmetric figure (convex or not) is a bisector of it.

Thus we have the following corollary.

CoRollary. Let $K$ be a compact convex figure. The following four statement are equivalent:

(a) the point $P$ through which three bisectors of $K$ pass is unique,

(b) all bisectors of $K$ are concurrent in $P$;

(c) there exists a point $P$ such that any line through it is a bisector of $K$;

(d) $K$ is a centrally symmetric figure with $P$ as its centre.

REMARKS. 1. K. Zarankiewicz appears to have proved a similar theorem for convex figures (see [3], page 264, note 10). Our result is in a more general setting, and is, surprisingly, quite strong. The author believes that his proof is different from that given by Zarankiewicz.

2. A stronger statement of the theorem is not possible, in the sense that out of the four statements (a), (b), (c), (d) mentioned in the corollary, it is not true in general that (c) implies (b), (since a bisector in a direction need not be unique). Also mass-distributions can be constructed easily for which (a) is true but (d) is not. (I am grateful to the referee for bringing to my notice an example where (a), (b), (c) are true but (d) is not).

3. Consider the set of points through which three bisectors pass. Very little is known about this set (see, however, [2]).

Acknowledgement. I am thankful to the referee for his helpful suggestions, and for having pointed out the reference [2].

\section{REFERENCES}

1. R.C. Buck and E.F. Buck, Equipartition of convex sets, Math. Mag. $22(1948 / 49)$, 
195-198.

2. M. Goldberg, On area-bisectors of plane convex sets, Amer. Math. Monthly 70 (1963), 529-531.

3. B. Grünbaum, Measures of symmetry for convex sets, Proceedings of symposia in Pure Maths. Convexity, 7 (1963), 233-270.

4. I.M. Yaglom and V.G. Boltyanskii, Convex Figures, Holt, Rinehart and Winston, New York, 1961.

INDIAN STATISTICAL INSTITUTE 



\section{PACIFIC JOURNAL OF MATHEMATICS}

EDITORS

H. SAMELSON

Stanford University

Stanford, California

R. M. BLUMENTHAL

University of Washington

Seattle, Washington 98105
*J. DUGUNDJI

University of Southern California

Los Angeles, California 90007

RICHARD ARENS

University of California

Los Angeles, California 90024

\section{ASSOCIATE EDITORS}

E. F. BECKENBACH

B. H. NeumanN

F. WOLF

K. YosidA

\section{SUPPORTING INSTITUTIONS}

UNIVERSITY OF BRITISH COLUMBIA

CALIFORNIA INSTITUTE OF TECHNOLOGY

UNIVERSITY OF CALIFORNIA

MONTANA STATE UNIVERSITY

UNIVERSITY OF NEVADA

NEW MEXICO STATE UNIVERSITY

OREGON STATE UNIVERSITY

UNIVERSITY OF OREGON

OSAKA UNIVERSITY

UNIVERSITY OF SOUTHERN CALIFORNIA
STANFORD UNIVERSITY

UNIVERSITY OF TOKYO

UNIVERSITY OF UTAH

WASHINGTON STATE UNIVERSITY

UNIVERSITY OF WASHINGTON

AMERICAN MATHEMATICAL SOCIETY CHEVRON RESEARCH CORPORATION

TRW SYSTEMS

NAVAL ORDNANCE TEST STATION 


\section{Pacific Journal of Mathematics}

\section{Vol. 16, No. $1 \quad$ November, 1966}

Larry Armijo, Minimization of functions having Lipschitz continuous first

partial derivatives ............................... 1

Edward Martin Bolger and William Leonard Harkness, Some

characterizations of exponential-type distributions.............. 5

James Russell Brown, Approximation theorems for Markov operators ...... 13

Doyle Otis Cutler, Quasi-isomorphism for infinite Abelian p-groups ...... 25

Charles M. Glennie, Some identities valid in special Jordan algebras but not valid in all Jordan algebras .......................... 47

Thomas William Hungerford, A description of $\operatorname{Mult}_{i}\left(A^{1}, \cdots, A^{n}\right)$ by

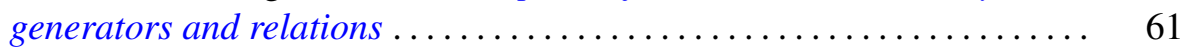

James Henry Jordan, The distribution of cubic and quintic non-residues ....

Junius Colby Kegley, Convexity with respect to Euler-Lagrange differential operators................................... 87

Tilla Weinstein, On the determination of conformal imbedding ......... 113

Paul Jacob Koosis, On the spectral analysis of bounded functions ........ 121

Jean-Pierre Kahane, On the construction of certain bounded continuous functions ................................... 129

V. V. Menon, A theorem on partitions of mass-distribution ........... 133

Ronald C. Mullin, The enumeration of Hamiltonian polygons in triangular

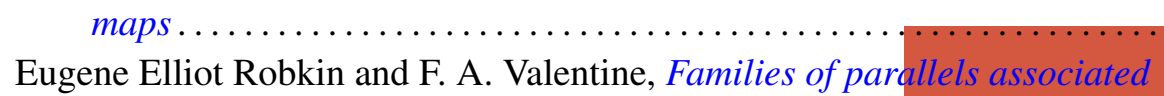

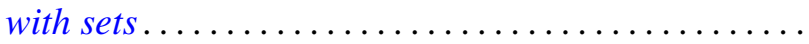

Melvin Rosenfeld, Commutative F-algebras

A. Seidenberg, Derivations and integral closure

S. Verblunsky, On the stability of the set of exponents of a Cauchy

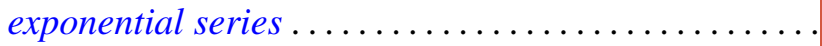

Herbert Walum, Some averages of character sums 